\title{
THE $\left(\phi^{2 n}\right)_{2}$ FIELD HAMILTONIAN FOR COMPLEX COUPLING CONSTANT $\left({ }^{1}\right)$
}

\author{
BY \\ LON ROSEN AND BARRY SIMON
}

\begin{abstract}
We consider hamiltonians $H_{\beta}=H_{0}+\beta H_{I}(g)$, where $H_{0}$ is the hamiltonian of a free Bose field $\phi(x)$ of mass $m>0$ in two-dimensional space-time, $H_{I}(g)$ $=\int g(x): P(\phi(x)): d x$ where $g \geqq 0$ is a spatial cutoff and $P$ is an arbitrary polynomial which is bounded below, and the coupling constant $\beta$ is in the cut plane, i.e. $\beta \neq$ negative real. We show that $H_{\beta}$ generates a semigroup with hypercontractive properties and satisfies higher order estimates of the form $\left\|H_{0} N^{r} R_{\beta}^{s}\right\|<\infty$, where $N$ is the number operator, $R_{\beta}=\left(H_{\beta}+b\right)^{-1}, r$ a positive integer, and $\beta, s$, and $b$ are suitably chosen. For any $0 \leqq \Theta<\pi, R_{\beta}$ converges in norm to $R_{0}$ as $|\beta| \rightarrow 0$ with $|\arg \beta| \leqq \Theta$. Finally we discuss applications of these results and establish asymptotic series and Borel summability for various objects in the real $\beta$ theory.
\end{abstract}

1. Introduction. Let $\phi(x)$ be a free Bose field of mass $m>0$ in two space-time dimensions, and let $H_{0}$ be the corresponding hamiltonian: $H_{0}=\int \mu(k) a^{*}(k) a(k) d k$, where $\mu(k)=\left(m^{2}+k^{2}\right)^{1 / 2}$. Define $H_{I}(g)=\int g(x): P(\phi(x)): d x$, where $g \in C_{0}^{\infty}(\mathscr{R})$ is real-valued and $P(y)=b_{2 n} y^{2 n}+b_{2 n-1} y^{2 n-1}+\cdots+b_{0}$ is a polynomial which is bounded below, i.e. $b_{2 n}>0$. It can be shown [2] that $H_{I}(g)$ is a selfadjoint operator on a suitable domain. We shall also assume that $g(x) \geqq 0$ so that $H_{I}(g)$ is formally positive. Although the Wick ordering destroys this positivity, $H_{I}(g)$ has an "almost positive" character made precise by Nelson's remark [7] that if $\operatorname{Re} \beta \geqq 0$ then $\left(\Omega_{0}, \exp \left(-\beta H_{I}(g)\right) \Omega_{0}\right)$ is finite, where $\Omega_{0}$ is the Fock vacuum. The hamiltonian

$$
H_{\beta}=H_{0}+\beta H_{I}(g)
$$

with positive coupling constant $\beta$ plays a central role in the construction of the $P(\phi)_{2}$ field theory without cutoffs. $H_{\beta}$ has been extensively studied and proved to be selfadjoint [1], [8], [9], [10], [13]. (See [2] for further references and a summary of recent progress.)

Our purpose in this note is to study $H_{\beta}$ for complex $\beta$ in the cut plane, i.e. $\beta$ not equal to a negative real. (Even the nonrelativistic analogue, $p^{2}+x^{2}+\beta x^{4}$, is misbehaved when $\beta$ is a negative real [12].) Previous to our discussion the following results have been obtained for complex coupling constant by B. Simon and $\mathbf{R}$. Hoegh-Krohn:

Received by the editors May 5, 1971.

AMS 1970 subject classifications. Primary 81A18; Secondary 47A55.

Key words and phrases. Boson fields, polynomial interaction, hamiltonian, vacuum expectation values, coupling constant analyticity, asymptotic series, Borel summability.

( $\left.{ }^{1}\right)$ Research partially supported by NSF GP-15735.

Copyright (C) 1972, American Mathematical Society 
(a) [13] $H_{\beta}$ is a sectorial operator with numerical range contained in a sector of opening angle not greater than $\arg \beta$;

(b) [13] As $|\beta| \rightarrow 0$ with $|\arg \beta| \leqq \pi / 2-\varepsilon,\left(H_{\beta}-z\right)^{-1} \rightarrow\left(H_{0}-z\right)^{-1}$ in norm, for $z \in \operatorname{res}\left(H_{0}\right)$;

(c) [11] In the special case of $P(y)=y^{4}$, the resolvent convergence of (b) holds for $|\arg \beta| \leqq \pi-\varepsilon$.

We work with the two equivalent representations of the $P(\phi)$ theory (cf. [2]). The first is Fock space $\mathscr{F}=\sum_{n=0}^{\infty} \oplus \mathscr{F}^{n}$ consisting of a direct sum of $n$-particle spaces; on $\mathscr{F}, H_{0}$ is a multiplication operator. The second is " $Q$-space" or $L^{2}(M, \mu)$ where $\mu$ is a probability measure on $M$; here the fields $\phi(x)$ and hence $H_{I}(g)$ are multiplication operators. Our approach is to exploit the "smoothing" properties of $H_{0}$ and $H_{\beta}$ in each of these representations. On $\mathscr{F}, H \equiv H_{\beta}$ is smoothing in the sense that for sufficiently large $b>0,(H+b)^{-1}$ maps $D\left(N^{r}\right)$ into $D\left(N^{r+1}\right)$,

$$
\left\|N^{r+1}(H+b)^{-1}(N+1)^{-r}\right\|<\infty \text {. }
$$

Here $N$ is the number operator: $N=n$ on $\mathscr{F}^{n}$. On $L^{2}(M, \mu), H_{0}$ is smoothing in the sense that, for sufficiently large $t>0$,

$$
\exp \left(-t H_{0}\right): L^{2}(M, \mu) \rightarrow L^{4}(M, \mu)
$$

and similarly for $e^{-t H}$ if $t$ is suitably chosen.

Higher order estimates, typified by (1.2), have been proved in the case of real $\beta$ in [9]; the method of hypercontractive semigroups whose starting point is (1.3) has been studied in [10] and [13], primarily in the case of real $\beta$. In $\S 2$ we sketch an extension of the latter method to the $P(\phi)$ theory with $\beta$ in the cut plane; this allows us in particular to obtain the result (b) above for $|\arg \beta| \leqq \pi-\varepsilon$. In $\S 3$ we show that the higher order estimates carry over to the case of complex $\beta$ with the most complete results being obtained for the $\phi^{4}$ theory.

Our reason for studying the complex $\beta$ theory is not that we feel it is intrinsically interesting but rather that it can be used to study properties of the analytic continuation of various objects in the real $\beta$ theory. For instance, properties (a)-(c) above have already been used to prove [13] that for the $P(\phi)$ theory the ground state energy and the equal time vacuum expectation values (VEV) of the fields have asymptotic series in $|\arg \beta| \leqq \pi / 2-\varepsilon$; and [11] that for the $\phi^{4}$ theory this series for the ground state energy is Borel summable. In $\S \S 4$ and 5 we similarly consider applications of the results of $\$ \S 2$ and 3 . Thus we extend the region of validity of the various asymptotic series to $|\arg \beta| \leqq \pi-\varepsilon$ and we prove Borel summability of the equal time VEV's.

2. Hypercontractive semigroup techniques. We first recall the definition and basic properties of a hypercontractive semigroup (see [13]). Let $H_{0} \geqq 0$ be a selfadjoint operator on $L^{2}(M, \mu)$ where $\mu$ is a probability measure on $M$. The set of operators $\left\{\exp \left(-t H_{0}\right) \mid t \geqq 0\right\}$ is a hypercontractive semigroup if $\exp \left(-t H_{0}\right)$ is a 
contraction on $L^{1}(M, \mu)$ for all $t \geqq 0$ and if, for some $T>0$, $\exp \left(-T H_{0}\right)$ is a contraction from $L^{2}(M, \mu)$ to $L^{4}(M, \mu)$. From these two assumptions it follows by interpolation that in fact $\exp \left(-t H_{0}\right)$ is a contraction on $L^{p}$ for all $1 \leqq p \leqq \infty$ and $t \geqq 0$,

$$
\left\|\exp \left(-t H_{0}\right) \psi\right\|_{p} \leqq\|\psi\|_{p} .
$$

Moreover for any $p>1$ and $q<\infty$ there is a $T>0$ such that for $t \geqq T$

$$
\left\|\exp \left(-t H_{0}\right) \psi\right\|_{q} \leqq\|\psi\|_{p} .
$$

This smoothing property holds as well for small $t$ provided that $q$ is not much larger than $p$ in the sense that $q-p=O(t)$.

Consider now "complex time" $z$ with fixed argument $\theta$,

$$
z=t e^{i \theta} ; \quad t \geqq 0 \text { and }|\theta|<\pi / 2 .
$$

Regarded as a semigroup in $t=|z|, \exp \left(-z H_{0}\right)$ also enjoys the above properties with some slight modifications. For by applying the Stein Interpolation Theorem to $f(s)=\exp \left(-t H_{0} e^{i s \pi / 2}\right)$ we find that $\exp \left(-z H_{0}\right)$ is a contraction on $L^{p}$ provided that (see Stein [14])

$$
(1-|\theta| / \pi)^{-1} \leqq p \leqq \pi /|\theta| .
$$

Since $\exp \left(-z H_{0}\right)=\exp \left(-t \cos \theta H_{0}\right) \exp \left(-i t \sin \theta H_{0}\right)$ we see from (2.2) that, for any $q<\infty$ and $p=2$, there is a $T$ such that

$$
\left\|\exp \left(-z H_{0}\right) \psi\right\|_{q} \leqq\|\psi\|_{p}
$$

when $t \cos \theta \geqq T$. By duality and interpolation we can extend (2.5) to the case of arbitrary $p>1$, as well as to the case of small $|z|$ provided that $p$ satisfies (2.4) and $q$ is not much larger than $p$.

It is thus possible to apply the methods developed for the perturbation of hypercontractive semigroups [10], [13]: If $H_{0}$ is the generator of a hypercontractive semigroup, we consider perturbations $H_{0}+V$ where

$$
V \in L^{p}(M, \mu) \text { for all } p<\infty
$$

and

$$
e^{-z V} \in L^{1}(M, \mu) \text { for all } t=|z| \geqq 0, \arg z=\theta .
$$

Define $H$ to be the closure of the sum

$$
H=\left.\left(H_{0}+V\right)\right|_{\mathscr{D}} ^{-}
$$

where $\mathscr{D}=D\left(H_{0}\right) \cap D(V)$. We can then prove

THEOREM 2.1. Under the above assumptions on $H_{0}, V$, and $z, H$ is the generator of a semigroup $e^{-z H}$ on $L^{q}(M, \mu)$ which is strongly continuous and exponentially bounded in $|z|$ provided that $q$ is in the interval

$$
I(\theta)=\left((1-|\theta| / \pi)^{-1}, \pi /|\theta|\right),
$$


i.e. there is a constant $a>0$ dependent on $\theta$ and $q$ such that

$$
\left\|e^{-z H} \psi\right\|_{q} \leqq a^{|z|}\|\psi\|_{q}
$$

We now specialize to the quantum field case with $V=\beta H_{I}(g)$, where the coupling constant $\beta$ is in the cut plane, $|\arg \beta|<\pi$. It is known [2] that $V$ satisfies conditions (2.6) and (2.7) provided that $\operatorname{Re}(z \beta) \geqq 0$. Thus $\exp \left(-z H_{\beta}\right)$ satisfies $(2.10)$ when $z=t e^{i \theta}$ lies in the cone

$$
C=\{z|| \theta|<\pi / 2,| \theta+\arg \beta \mid \leqq \pi / 2\} .
$$

It follows from standard semigroup theory that the $L^{2}$ spectrum of $H_{\beta}$ lies in the cone $C^{\prime}$ dual to $C$ with vertex at $x=-\inf \log a$,

$$
C^{\prime}=\left\{z^{\prime} \mid \operatorname{Re}\left(\left(z^{\prime}-x\right) z\right)>0, \text { for all } z \in C\right\} .
$$

This result we recognize as being essentially property (a) of $\S 1$ which was established more directly in [13].

We collect here some additional properties of the hamiltonian $H$, most of which are also valid in the abstract hypercontractive setting (the argument $\theta$ of $z$ is regarded as being fixed in $(-\pi / 2, \pi / 2))$ :

(i) (Smoothing property.) Suppose that $p, q$ lie in the interval $I(\theta)$ of (2.9). Then there is a $T>0$ such that if $|z| \geqq T, e^{-z H}$ is a bounded operator from $L^{p}$ to $L^{q}$. Given $p$, the same is true for small $t>0$ provided that $q-p=O(t)$.

(ii) (Continuous dependence on $V$.) Suppose that $\left\{V_{j}\right\}$ is a sequence of functions on $M$ such that for each $p<\infty$ and $t=|z| \geqq 0$

$$
\lim _{j \rightarrow \infty}\left\|V_{j}-V\right\|_{p}=0 \text { and } \sup _{j}\left\|\exp \left(-z V_{j}\right)\right\|_{1}<\infty .
$$

Define $H_{j}=H_{0}+V_{j}$. Then for all $t \geqq 0$ and $p, q \in I(\theta)$,

$$
\exp \left(-z H_{j}\right) \rightarrow \exp (-z H)
$$

in the sense of norm convergence as operators on $L^{p}$, and, for sufficiently large $t$, as operators from $L^{p}$ to $L^{q}$.

(iii) (Definition of resolvent.) Let $p \in I(\theta)$. For $\operatorname{Re}\left(\lambda e^{i \theta}\right)$ sufficiently negative, $R(\lambda)=(H-\lambda)^{-1}$ is a bounded operator from $L^{p}$ to $L^{p}$.

(iv) (Resolvent convergence.) Suppose $\left\{V_{j}\right\}$ is a sequence as in (ii) and let $p \in I(\theta)$. For $\operatorname{Re}\left(\lambda e^{i \theta}\right)$ sufficiently negative $R_{j}(\lambda)=\left(H_{j}-\lambda\right)^{-1}$ converges to $R(\lambda)$ in the sense of norm convergence as operators on $L^{p}$.

(v) (Zero coupling limit.) Let $p \in I(\theta)$ and suppose that $\left\{\beta_{j}\right\}$ is a sequence in the cut plane converging to 0 such that $\operatorname{Re}\left(e^{i \theta} \beta_{j}\right) \geqq 0$. Define $H_{j}=H_{0}+\beta_{j} H_{I}(g)$. Then for all $t \geqq 0$ and $\operatorname{Re}\left(\lambda e^{i \theta}\right)$ sufficiently negative $\exp \left(-z H_{j}\right) \rightarrow \exp \left(-z H_{0}\right)$ and $\left(H_{j}-\lambda\right)^{-1} \rightarrow\left(H_{0}-\lambda\right)^{-1}$ in the sense of norm convergence as operators on $L^{p}$.

(vi) (Stability of the vacuum.) Suppose that $0<|\lambda|<m$ where $m$ is the bare mass. Then there is a $B$ such that $\left(H_{0}+\beta H_{I}-\lambda\right)^{-1}$ is bounded if $|\beta| \leqq B$. This bound is uniform for $\beta$ and $\lambda$ satisfying $|\lambda|=\mu,|\beta| \leqq B,|\arg \beta| \leqq A$ where $0<\mu<m$ and $A<\pi$. In fact, any $\lambda \in \operatorname{res}\left(H_{0}\right)$ is also in res $\left(H_{\beta}\right)$ for $|\beta|$ sufficiently small. 
Properties (i)-(vi) follow from hypercontractive semigroup techniques as in [13]. (i) is a consequence of the Trotter Product Formula and the smoothing property (2.5) of $\exp \left(-z H_{0}\right)$. (i) leads to (ii) by means of Duhamel's formula. The corresponding results (iii) and (iv) for the resolvent follow from taking Laplace transforms. (v) is a special case of (ii) and (iv), and (vi) follows from (v) by general stability theorems (cf. [4, p. 206]). We remark that the proof of (v) that we have sketched here is very different from that used to conclude the same result in the special case of $\phi^{4}$.

3. Higher order estimates. In this section we prove higher order estimates of the form

$$
\left\|(N+1)^{r+1} R_{\beta}(-b)(N+1)^{-r}\right\|<\infty .
$$

Here $N$ is the number operator, $r \geqq 0$ an integer, $R_{\beta}(\lambda)=\left(H_{0}+\beta H_{I}(g)-\lambda\right)^{-1}$, and $b>0$ a suitably large constant. Our results are incomplete inasmuch as we can prove (3.1) in the case of $P(\phi)$ only for $|\arg \beta|<\pi / 4$, but in the special case of $\phi^{4}$ for $\beta$ in the cut plane with $|\beta|$ small.

Define the domain

$$
\begin{array}{r}
D=\left\{\Psi \mid \Psi=\left(\Psi_{0}, \Psi_{1}, \ldots\right) \in \mathscr{F} ; \Psi_{n} \in C_{0}^{\infty}\left(\mathscr{R}^{n}\right) ;\right. \\
\left.\Psi_{n}=0 \text { for sufficiently large } n\right\} .
\end{array}
$$

$D$ provides a domain of "nice" vectors on which the following operator calculations are well defined. But in order to extend the resulting operator inequalities to domains larger than $D$ we should like to know that $D$ is a core for $(N+1)^{\alpha}(H-\lambda)$; here $\alpha$ is real and $\lambda \in$ res $(H)$. We have been unable to prove this (although our original manuscript did contain a "proof" based on a misuse of Nelson's analytic vector theorem). Consequently the proofs of Theorem 3.1 and Lemma 3.4 below are only formally true. At the end of this section we indicate how to overcome this difficulty by introducing a momentum cutoff in $H$.

THEOREM 3.1. Assume that $|\arg \beta|<\pi / 4$, and let $r \geqq 0$ be an integer. Then for $b>0$ sufficiently large, we have the estimates (3.1) and (3.2),

$$
\left\|(N+1)^{r+1} R_{\beta}(-b)^{r+1}\right\|<\infty .
$$

Proof. Since the proof differs only slightly from that for the case of $\beta>0$, we sketch the details (cf. $[9, \S 4])$. The main steps are as follows:

(i) The estimate (3.2) follows by induction from (3.1). Assuming that $D$ is a core for $(N+1)^{r}(H+b)$, it is sufficient to take $\Psi \in D^{\prime}=(H+b) D$ and to prove (3.1) in the form

$$
\left\|(N+1)^{r+1} R_{\beta}(-b) \Psi\right\| \leqq \text { const. }\left\|(N+1)^{r} \Psi\right\| .
$$

(ii) Since $R_{\beta} \Psi \in D$ we can rewrite $\left\|(N+1)^{r+1} R_{\beta} \Psi\right\|$ in terms of integrals involving products of annihilation operators. 
(iii) The annihilation operators are "pulled through" the resolvent $R_{\beta}$ by means of the Pull Through Formula [9, Proposition 4.5].

(iv) The resulting terms are estimated and are shown upon integration to be dominated by $\left\|(N+1)^{r} \Psi\right\|$. The basic estimate involved is of the form

$$
\left\|H_{0}^{1 / 2} R_{\beta}(-b) Q R_{\beta}(-b) H_{0}^{1 / 2}\right\|<\infty
$$

where $Q$ is a polynomial in the field of degree less than that of the interaction $P(\phi)$.

We indicate the proof of (3.4). Let $\beta=x+i y$ where $|y|<x$. When $y=0$, (3.4) follows at once from the inequalities

$$
\left\|H_{0}^{1 / 2} R_{x}(-b)^{1 / 2}\right\|<\infty
$$

and

$$
\left\|R_{x}(-b)^{1 / 2} Q R_{x}(-b)^{1 / 2}\right\|<\infty
$$

for sufficiently large $b$. Both (3.5) and (3.6) amount to restatements of the semiboundedness of $H_{0}+\tilde{x} \tilde{H}_{I}$ for suitable $\tilde{H}_{I}$ of $P(\phi)$ form and suitable real $\tilde{x}$.

When $y \neq 0$, (3.4) remains valid since

$$
R_{\beta}=R_{x}^{1 / 2} B R_{x}^{1 / 2}
$$

where $B$ is a bounded operator. To prove (3.7) we note that

$$
x H_{I} \leqq H_{0}+x H_{1}+b
$$

so that

$$
\left\|R_{x}(-b)^{1 / 2} y H_{I} R_{x}(-b)^{1 / 2}\right\| \leqq|y| / x<1 .
$$

Taking $B=\left(1+R_{x}(-b)^{1 / 2} i y H_{I} R_{x}(-b)^{1 / 2}\right)^{-1}$, we verify (3.7) by the expansion

$$
R_{\beta}=R_{x}^{1 / 2} \sum_{j=0}^{\infty}\left(-R_{x}^{1 / 2} i y H_{I} R_{x}^{1 / 2}\right)^{j} R_{x}^{1 / 2}
$$

Corollary 3.2. Assume that $|\arg \beta|<\pi / 4$ and let $r \geqq 0$ be an integer. Then for $b>0$ sufficiently large

$$
\left\|H_{0} N^{r} R_{\beta}(-b)^{n+r}\right\|<\infty
$$

where $2 n$ is the degree of the interaction $P(\phi)$.

REMARK. In the special case of $P(\phi)=\phi^{4}$ it is possible to take $n=1$ in (3.8) while in the general case this remains an open question.

Proof. By a standard $N$-estimate (cf. [2])

$$
\left\|(N+1)^{-\alpha} H_{I}(g)(N+1)^{-\beta}\right\|<\infty
$$

provided $\alpha+\beta \geqq n$. It follows from (3.2) that $N^{r} H_{I} R_{\beta}^{n+r}$ is bounded; so is $N^{r}\left(H_{0}+\beta H_{I}\right) R_{\beta}^{r+1}$. Since $H_{0} N^{r} R_{\beta}^{n+r}=N^{r}\left(H_{0}+\beta H_{I}\right) R_{\beta}^{n+r}-\beta N^{r} H_{I} R_{\beta}^{n+r}$, we obtain (3.8). 
Here we have used the facts that $N \leqq$ const. $H_{0}$, and $(N+1)^{i} \leqq(N+1)^{j}$ if $i \leqq j$. Assuming that $D^{\prime}$ is a core for $(N+1)^{r}$, we obtain (3.17) from (3.20) by taking closures.

We now turn to the proof of (3.18) on $D \times D$. Set $\beta=x+i y$. Then

$$
\begin{aligned}
\left(H^{*}+1\right)(N+1)^{2 r}(H+1)= & \left(H_{0}+1\right)^{2}(N+1)^{2 r}+|\beta|^{2} H_{I} N^{2 r} H_{I} \\
& +x\left(H_{I}(N+1)^{2 r}\left(H_{0}+1\right)+\left(H_{0}+1\right)(N+1)^{2 r} H_{I}\right) \\
& +i y\left[\left(H_{0}+1\right)(N+1)^{2 r}, H_{I}\right] \\
= & x|/| \beta \mid\left(H_{0}+1+\operatorname{sgn} x|\beta| H_{I}\right)(N+1)^{2 r}\left(H_{0}+1+\operatorname{sgn} x|\beta| H_{I}\right) \\
& +(1-|x| /|\beta|)\left(\left(H_{0}+1\right)^{2}(N+1)^{2 r}+|\beta|^{2} H_{I}(N+1)^{2 r} H_{I}\right) \\
& +i y\left[\left(H_{0}+1\right)(N+1)^{2 r}, H_{I}\right] \\
\geqq & (1-|x| /|\beta|)\left(H_{0}+1\right)^{2}(N+1)^{2 r}+i y\left[\left(H_{0}+1\right)(N+1)^{2 r}, H_{I}\right] \\
\geqq & \text { const. }\left(H_{0}+1\right)^{2}(N+1)^{2 r},
\end{aligned}
$$

for sufficiently small $B$. In the last step we have arranged that $|y|$ is small and dominated the commutator by (3.10).

COROLLARY 3.5. Under the hypotheses of the lemma, there is a B such that

$$
\left\|\left(H_{0}+1\right)(N+1)^{r} R_{\beta}(\lambda)^{r+1}\right\|<\infty
$$

with the bound uniform in $\beta$ which satisfy $|\beta| \leqq B$ and $\varepsilon \leqq|\arg \beta| \leqq \pi-\varepsilon$, and $\lambda$ in bounded subsets of $C$.

Proof. The corollary follows from the lemma by induction.

Combining Theorem 3.1 and Corollary 3.5 we can thus state for the $\phi^{4}$ theory:

THEOREM 3.6. Let $H_{I}=: \phi^{4}(g): \lambda \in \operatorname{res}\left(H_{0}\right)$, and suppose that $\varepsilon>0$ and the integer $r \geqq 0$ are given. There is a positive constant $B$ such that if $|\beta| \leqq B$ and $|\arg \beta|$ $\leqq \pi-\varepsilon$, then $\lambda \in \operatorname{res}\left(H_{\beta}\right)$,

$$
\begin{gathered}
\left\|(N+1)^{r+1} R_{\beta}(\lambda)(N+1)^{-r}\right\|<\infty, \\
\left\|(N+1)^{r+1} R_{\beta}(\lambda)^{r+1}\right\|<\infty,
\end{gathered}
$$

and

$$
\left\|\left(H_{0}+1\right)(N+1)^{r} R_{\beta}(\lambda)^{r+2}\right\|<\infty
$$

where the bounds are uniform in $\beta$.

Proof. As noted in (vi) of $\S 2, \lambda \in \operatorname{res}\left(H_{\beta}\right)$ for $B$ sufficiently small. Once we verify (3.21), then (3.22) and (3.23) follow as in Theorem 3.1 and Corollary 3.2. In fact (cf. (3.1) and (3.17)) we have already proved (3.21) except that for $|\arg \beta| \leqq \varepsilon$ we have restricted $\lambda$ to be a large negative real (assume $\varepsilon<\pi / 4$ ).

It remains to extend (3.21) to any $\lambda \in \operatorname{res}\left(H_{0}\right)$. To this end, we first show that, for $|\beta|$ small,

$$
\left\|(N+1)^{r} R_{\beta}(\lambda)(N+1)^{-r}\right\|<\infty .
$$


If we regard $\mathscr{H}_{r}=D\left((N+1)^{r}\right) \subset \mathscr{F}$ as a Hilbert space with norm $\|\Psi\|_{r}=\left\|(N+1)^{r} \Psi\right\|$, then (3.24) can be interpreted as the statement that $R_{\beta}(\lambda)$ is bounded on $\mathscr{H}_{r}$. We know that (3.24) is valid for $\lambda=-b$ sufficiently negative and for $\beta=0$ since $H_{0}$ commutes with $N$. Moreover $R_{\beta}(-b) \rightarrow R_{0}(-b)$ in norm on $\mathscr{H}_{r}$ as $|\beta| \rightarrow 0$; for we calculate that

$$
\begin{aligned}
& \left\|(N+1)^{r}\left[R_{\beta}(-b)-R_{0}(-b)\right](N+1)^{-r}\right\| \\
& \quad=|\beta|\left\|(N+1)^{r} R_{\beta}(-b) H_{I} R_{0}(-b)(N+1)^{-r}\right\| \\
& \quad \leqq|\beta|\left\|(N+1)^{r} R_{\beta}(-b)(N+1)^{-r+1}\right\| \cdot\left\|(N+1)^{r-1} H_{I}\left(H_{0}+b\right)^{-1}(N+1)^{-r}\right\| .
\end{aligned}
$$

By (3.1) and (3.9) we see that (3.25) is $O(|\beta|)$. We conclude by the cited stability theorem [4, p. 206] that $\lambda \in \operatorname{res}\left(H_{\beta}\left\lceil\mathscr{H}_{r}\right)\right.$ for $|\beta|$ sufficiently small; that is, (3.24) holds.

But

$$
\begin{aligned}
(N+1)^{r+1} & R_{\beta}(\lambda)(N+1)^{-r} \\
& =(N+1)^{r+1} R_{\beta}(-b)(N+1)^{-r}\left[1+(b+\lambda)(N+1)^{r} R_{\beta}(\lambda)(N+1)^{-r}\right] .
\end{aligned}
$$

According to this identity, (3.1) and (3.24) yield the desired conclusion.

REMARKS. With a good deal more work involving the method of double commutators it is possible to prove (3.23) with the power $R_{\beta}^{r+1}$.

We have been unable to prove these higher order estimates for the general $P(\phi)$ theory when $|\arg \beta| \geqq \pi / 4$. Perhaps we should explain why neither the "pull through" method of Theorem 3.1 nor the commutator method of Lemma 3.4 works in this case. In the first method, operator inequalities like (3.4) could be established even when $\beta$ is in the cut plane and $R_{\beta}$ is not selfadjoint, provided that we could prove that operators of the form $H_{\beta}^{1 / 2}\left(R_{\beta}^{*}\right)^{1 / 2}$ were bounded. However there exist sectorial operators for which $A^{1 / 2}\left(A^{*}\right)^{-1 / 2}$ is not bounded [5], [6], and we are unable to prove that $H_{\beta}$ with $\operatorname{Im} \beta \neq 0$ does not fall into this pathological class. The virtue of the commutator method or the double commutator method referred to above is that each commutator of $N$ (or $H_{0}$ ) with $H_{I}$ reduces the "number singularity" of $H_{I}$ by one. In the case of $\phi^{4}, H_{I}$ has a number singularity of order only $N^{2}$ and thus a commutator can be dominated by the other terms that occur as in (3.10). This is no longer possible when $\operatorname{deg} P \geqq 6$. Experience with the nonrelativistic case [12] suggests one might be able to bound the commutator with the $H_{I}^{2}$ term but no effective way of utilizing $H_{I}^{2}$ has been found.

We remark that the higher order estimates provide yet another proof of resolvent convergence as $|\beta| \rightarrow 0$ (at least in the region of validity in the $\beta$ plane of the estimates). For $\phi^{4}, R_{\beta}(-b)-R_{0}(-b)=O(|\beta|)$ by (3.25) with $r=0$. We can similarly show that, for $\phi^{2 n}, R_{\beta}(-b)^{n-1}-R_{0}(-b)^{n-1}=O(|\beta|)$ for $|\arg \beta|<\pi / 4$.

Finally, we settle the domain problem that arose in the previous proofs. Let $H_{I, K}$ be a momentum cutoff version of $H_{I}$, obtained for instance by truncating the momentum integrals (3.11) occurring in $H_{I}$ to have domain of integration $\left|k_{i}\right| \leqq K$. Define $H_{K, \beta}=H_{0}+\beta H_{I, K}$. 
We now specialize to the $\phi^{4}$ model and extend (3.8) to $\beta$ in the cut plane (with $|\beta|$ small). It is a curious fact that the proof of (3.8) is easier when $\operatorname{Im} \beta \neq 0$ than when $\beta>0$. We first need a technical estimate similar to that used by Glimm and Jaffe in [1].

LEMMA 3.3. Let $H_{I}=: \phi^{4}(g)$ : and let $r \geqq 0$ be an integer. Then

$$
\pm i\left[\left(H_{0}+1\right)(N+1)^{r}, H_{I}\right] \leqq \text { const. }\left(H_{0}+1\right)^{2}(N+1)^{r} \text {. }
$$

REMARK. It is for convenience only that we assume $H_{I}$ has no terms of lower degree or that we do not take $r$ to be any real number.

Proof. A standard calculation (cf. [2]) shows that $H_{I}=\sum_{j=0}^{4} W_{j}$ where

$$
W_{j}=\int d k_{1} \cdots d k_{4} w_{j}\left(k_{1}, \ldots, k_{4}\right) a^{*}\left(k_{1}\right) \cdots a^{*}\left(k_{j}\right) a\left(k_{j+1}\right) \cdots a\left(k_{4}\right)
$$

creates $j$ and destroys $(4-j)$ particles, and the kernel $w_{j} \in L^{2}\left(\mathscr{R}^{4}\right)$. It is thus sufficient to prove that, for $j=0, \ldots, 4$,

$$
A_{j}=\left(H_{0}+1\right)^{-1}(N+1)^{-r / 2}\left[\left(H_{0}+1\right)(N+1)^{r}, W_{j}\right]\left(H_{0}+1\right)^{-1}(N+1)^{-r / 2}
$$

is bounded. We show that $A_{j}$ is bounded as a bilinear form on $D \times D$ and the desired operator extension follows by the Riesz Representation Theorem. Moreover it is sufficient to prove that for $\Phi \in \mathscr{F}^{s+j} \cap D$, and $\Psi \in \mathscr{F}^{s+4-j} \cap D$

$$
\left|\left(\Phi, A_{j} \Psi\right)\right| \leqq a\|\Phi\|\|\Psi\|
$$

where $a$ is independent of $s$. For then, for $\theta, \eta \in D$,

$$
\begin{aligned}
\left|\left(\theta, A_{j} \eta\right)\right| & =\left|\sum_{s, s^{\prime}}\left(\theta_{s}, A_{j} \eta_{s^{\prime}}\right)\right|=\left|\sum_{s}\left(\theta_{s+j}, A_{j} \eta_{s+4-j}\right)\right| \\
& \leqq a \sum_{s}\left\|\theta_{s+j}\right\|\left\|\eta_{s+4-j}\right\| \leqq a\|\theta\|\|\eta\|
\end{aligned}
$$

by Schwarz's Inequality.

We further simplify the problem by writing $A_{j}=B_{j}+C_{j}$ and proving (3.12) separately for $B_{j}$ and $C_{j}$, where

and

$$
B_{j}=(N+1)^{-r / 2}\left[(N+1)^{r}, W_{j}\right](N+1)^{-r / 2}\left(H_{0}+1\right)^{-1}
$$

$$
C_{j}=\left(H_{0}+1\right)^{-1}(N+1)^{-r / 2}\left[H_{0}, W_{j}\right](N+1)^{r / 2}\left(H_{0}+1\right)^{-1} .
$$

With $\Phi, \Psi$ as above we compute that

where

$$
\left(\Phi, B_{j} \Psi\right)=b_{s}\left(\Phi,(N+1)^{-1} W_{j}\left(H_{0}+1\right)^{-1} \Psi\right)
$$

$$
b_{s}=(s+j+1)^{1-r / 2}\left[(s+j+1)^{r}-(s+5-j)^{r}\right](s+5-j)^{-r / 2} .
$$

But $b_{s}$ is bounded uniformly in $s$, and by an $N$-estimate, so is the operator $(N+1)^{-1} W_{j}\left(H_{0}+1\right)^{-1}$; hence,

$$
\left|\left(\Phi, B_{j} \Psi\right)\right| \leqq b\|\Phi\|\|\Psi\|
$$

where $b$ is independent of $s$. 
Similarly we calculate that

$$
\left(\Phi, C_{j} \Psi\right)=c_{s}\left(\Phi,\left(H_{0}+1\right)^{-1} W_{j}^{\prime}\left(H_{0}+1\right)^{-1} \Psi\right)
$$

where $c_{s}=(s+j+1)^{-r / 2}(s+5-j)^{r / 2}$ and $W_{j}^{\prime}$ has the form (3.11) but with kernel

$$
w_{j}^{\prime}\left(k_{1}, \ldots, k_{4}\right)=\left[\sum_{i=1}^{j} \mu\left(k_{i}\right)-\sum_{i=j+1}^{4} \mu\left(k_{i}\right)\right] w_{j}\left(k_{1}, \ldots, k_{4}\right) .
$$

By an $N_{\tau}$-estimate

$$
\left\|\left(H_{0}+1\right)^{-1} W_{j}^{\prime}\left(H_{0}+1\right)^{-1}\right\| \leqq \text { const. }\left\|E\left(k_{1}, \ldots, k_{4}\right)^{-1 / 2} w_{j}^{\prime}\left(k_{1}, \ldots, k_{4}\right)\right\|_{L^{2}}
$$

where $E\left(k_{1}, \ldots, k_{4}\right)=\max _{i \neq i^{\prime}} \mu\left(k_{i}\right) \mu\left(k_{i^{\prime}}\right)$. We estimate that

$$
E\left(k_{1}, \ldots, k_{4}\right)^{-1 / 2} \mu\left(k_{i}\right) \leqq \text { const. } \mu^{1 / 2}\left(k_{1}+\cdots+k_{4}\right) .
$$

Hence the norm in (3.15) is dominated by

$$
\text { const. }\left\|\mu^{1 / 2}\left(k_{1}+\cdots+k_{4}\right) w_{j}\left(k_{1}, \ldots, k_{4}\right)\right\|_{L^{2}}
$$

which is finite provided that $\hat{g}(k)|k|^{\varepsilon} \in L^{2}$ for $\varepsilon>0$ (cf. [8, Lemma 4.1]). Since $c_{s}$ is bounded uniformly in $s$ we see from (3.14) and (3.15) that

$$
\left|\left(\Phi, C_{j} \Psi\right)\right| \leqq c\|\Phi\|\|\Psi\|
$$

where $c$ is independent of $s$.

Combining (3.13) and (3.16) yields (3.12) and the lemma.

We next mimic a technique of [12] when $\operatorname{Im} \beta \neq 0$ :

LEMMA 3.4. Let $H_{I}=: \phi^{4}(g)$ :, and suppose that $\varepsilon>0$ and the integer $r \geqq 0$ are given. There is a constant $B$ such that if $|\beta| \leqq B, \varepsilon \leqq|\arg \beta| \leqq \pi-\varepsilon$, and $\lambda \in \operatorname{res}\left(H_{\beta}\right)$, then

$$
\left\|\left(H_{0}+1\right)(N+1)^{r} R_{\beta}(\lambda) \Psi\right\| \leqq c\left\|(N+1)^{r} \Psi\right\|
$$

where the constant $c$ is independent of $\beta$ and of $\lambda$ for $\lambda$ in bounded subsets of $C$.

Proof. It is sufficient to prove that on $D \times D$

$$
\left(H_{0}+1\right)^{2}(N+1)^{2 r} \leqq a^{2}\left(H^{*}+1\right)(N+1)^{2 r}(H+1)
$$

where the constant $a$ is independent of $\beta$ in the cited region. For then if $\Psi \in D$,

$$
\begin{aligned}
\left\|\left(H_{0}+1\right)(N+1)^{r} \Psi\right\| & \leqq a\left\|(N+1)^{r}(H+1) \Psi\right\| \\
& \leqq a\left\|(N+1)^{r}(H-\lambda) \Psi\right\|+a|\lambda-1|\left\|(N+1)^{r} \Psi\right\|
\end{aligned}
$$

by the triangle inequality. Setting $\Phi=(H-\lambda) \Psi \in D^{\prime} \equiv(H-\lambda) D$, we obtain from (3.19) by induction

$$
\begin{aligned}
\left\|\left(H_{0}+1\right)(N+1)^{r} R_{\beta}(\lambda) \Phi\right\| & \leqq \text { const. }\left\{\sum_{j=0}^{r}\left\|(N+1)^{j} \Phi\right\|+\left\|R_{\beta}(\lambda) \Phi\right\|\right\} \\
& \leqq \text { const. }\left\|(N+1)^{r} \Phi\right\| .
\end{aligned}
$$


LeMmA 3.7. Assume that $\beta \geqq 0$. For real $\alpha$ and $\lambda \in \operatorname{res}\left(H_{K, \beta}\right) D$ is a core for both $\left(H_{K, \beta}-\lambda\right)^{\alpha}$ and $(N+1)^{\alpha}\left(H_{K, \beta}-\lambda\right)$.

Proof. We sketch the proof which is based on the methods of [9]. By the Pull Through method we establish the estimate

$$
\left(H_{0}+1\right)^{j} \leqq a_{K}\left(H_{K, \beta}+b\right)^{j}
$$

where $a_{K}$ and $b$ are positive constants with $a_{K}$ depending on $K$. Actually the domain problem arises again in the proof of (3.26); thus it is necessary to pass to a further cutoff hamiltonian $H_{K, V, \beta}[9, \S 2]$ which is known to be essentially selfadjoint along with its powers on a nice domain and for which (3.26) can be definitely established with constants independent of the cutoff $V$. Then (3.26) is obtained by limits and the Principle of Cutoff Independence [9].

By $N$-estimates it is easy to prove a sort of inverse to (3.26):

$$
\left(H_{K, \beta}+b\right)^{j} \leqq c_{K}\left(H_{0}+1\right)^{n j} \text {. }
$$

Taken together (3.26) and (3.27) imply that

$$
D\left(H_{K, \beta}^{n j}\right) \subset D\left(H_{0}^{n j}\right) \subset D\left(H_{K, \beta}^{j}\right)
$$

and accordingly that $D\left(H_{0}^{n j}\right)$ is a core for $H_{K, \beta}^{j}$ since $D\left(H_{K, \beta}^{n j}\right)$ is. It follows at once from (3.27) that $H_{K, \beta}^{j}$ is essentially selfadjoint on $D$ since $H_{0}^{n j}$ is.

That $D$ is also a core for $(N+1)^{\alpha}\left(H_{K, \beta}-\lambda\right)$ is a consequence of (3.26) and the fact that $D$ is a core for $\left(H_{K, \beta}-\lambda\right)^{\alpha+1}$ (cf. [9, Lemma 4.9]).

It remains to extend Lemma 3.7 to nonreal $\beta$ in the region of interest, namely, $\{\beta|| \arg \beta \mid<\pi / 4\}$ for $P(\phi)$ and $\{\beta|| \arg \beta|<\pi,| \beta \mid<B\}$ for $\phi^{4}$. Call this region $\Omega$. For then we could prove the inequalities (3.3) and (3.17) for the cutoff theory, that is, with $R_{\beta}(\lambda)$ replaced by $R_{K, \beta}(\lambda)=\left(H_{K, \beta}-\lambda\right)^{-1}$ but with constants independent of $K$. When $K \rightarrow \infty, R_{K, \beta} \rightarrow R_{\beta}$ in norm by (iv) of $\S 2$ and [13, Lemma III.16], and thus we recover (3.3) and (3.17) by the Principle of Cutoff Independence.

To this end we note as in the proof of Lemma 3.7 that for $P(\phi)$ we can also prove an estimate like (3.17),

$$
\left\|(N+1)^{r}\left(H_{0}+1\right) \Psi\right\| \leqq c_{K}\left\|(N+1)^{r}\left(H_{K, \beta}+b\right) \Psi\right\|, \quad \Psi \in D,
$$

for $\beta \in \Omega$, where now the constant $c_{K}$ depends on $K$ but can be chosen independently of $\beta$ for $\beta$ in compact subsets of $\Omega$. It follows from (3.28) that

$$
\left\|(N+1)^{r} \beta H_{I, K} \Psi\right\|=\left\|(N+1)^{r}\left(H_{K, \beta}-H_{0}\right) \Psi\right\| \leqq d_{K}\left\|(N+1)^{r}\left(H_{K, \beta}+b\right) \Psi\right\|
$$

where $d_{K}$ is independent of $\beta$ in compact subsets of $\Omega$. Consequently, when $|\delta \beta|$ $<|\beta| / d_{K}$ we have

$$
\left\|\delta \beta(N+1)^{r} H_{I, K} \Psi\right\| \leqq a\left\|(N+1)^{r}\left(H_{K, \beta}+b\right) \Psi\right\|, \quad \Psi \in D,
$$

where $a<1$. That is, $\delta \beta(N+1)^{r} H_{I, K}$ is a small perturbation (in the sense of Kato [4]) of $(N+1)^{\gamma}\left(H_{K, \beta}+b\right)$, and if (3.29) holds on a core $C$ for $(N+1)^{\gamma}\left(H_{K, \beta}+b\right)$ then $C$ is also a core for $(N+1)^{r}\left(H_{K, \beta+\delta \beta}+b\right)$. 
It is now clear how to extend Lemma 3.7 by induction to any $\beta \in \Omega$. We reach $\beta$ by a sequence of points $\beta_{j}=|\beta| e^{i \theta_{g}}, j=0,1, \ldots, m$, where $\theta_{0}=0, \theta_{m}=\arg \beta$, and $\left|e^{i \theta_{j+1}}-e^{i \theta_{j}}\right|<1 / d_{K}$. By Lemma 3.7, $D$ is a core for $(N+1)^{\alpha}\left(H_{K, \beta_{0}}+b\right)$ and so by (3.29) it is also a core for $(N+1)^{\alpha}\left(H_{K, \beta_{1}}+b\right)$. Repeating this procedure completes the proofs of this section.

4. Asymptotic expansions. In this section we consider the asymptotic expansions of various stationary objects in terms of $\beta$ in the cut plane. For $\beta>0$ it is known [2] that $H_{\beta}=H_{0}+\beta H_{I}(g)$ has a unique ground state $\Omega_{\beta}$, normalized by $\left\|\Omega_{\beta}\right\|=1$ and $\left(\Omega_{\beta}, \Omega_{0}\right)>0$. Let $E_{\beta}=\left(\Omega_{\beta}, H_{\beta} \Omega_{\beta}\right)$ be the ground state energy and $P_{\beta}$ the projection onto $\Omega_{\beta}$. We shall also be concerned with equal time vacuum expectation values of products of fields,

$$
W_{\beta}=\left(\Omega_{\bar{\beta}}, \phi\left(h_{1}\right) \cdots \phi\left(h_{r}\right) \Omega_{\beta}\right),
$$

where $h_{j} \in L^{2}(\mathscr{R})$, and $\phi(h)=\int \phi(x, 0) h(x) d x$ is the time zero field.

For a fuller discussion of the following material see [13, §IV.3]. There it is shown that, by virtue of property (a) in the introduction, the operators $H_{\beta}$ form an analytic family of operators for $\beta$ in the cut plane. Here "analytic" means that the resolvents $R_{\beta}$ are norm differentiable in $\beta$; in fact, the family $H_{\beta}$ forms a selfadjoint analytic family of type $\left(B_{0}\right)$ in the sense of T. Kato [4, Chapter VII]. It follows that $\Omega_{\beta}$ and $E_{\beta}$ have an analytic continuation to a neighborhood of the positive real $\beta$-axis. Actually, we have analyticity in a larger region:

THEOREM 4.1. Let $\varepsilon>0$. Then there is a $B>0$ such that $E_{\beta}, \Omega_{\beta}$, and $W_{\beta}$ are analytic in $\{\beta|| \arg \beta|\leqq \pi-\varepsilon,| \beta \mid \leqq B\}$.

Proof. The analyticity of $E_{\beta}$ and $\Omega_{\beta}$ follows from the general theory of analytic families [4] and the norm resolvent convergence as $|\beta| \rightarrow 0$ of $\S 2$. As for $W_{\beta}$, we see from

$$
\frac{1}{\alpha-\beta}\left(W_{\alpha}-W_{\beta}\right)=\left(\frac{\Omega_{\bar{\alpha}}-\Omega_{\bar{\beta}}}{\bar{\alpha}-\bar{\beta}}, \phi\left(h_{1}\right) \cdots \phi\left(h_{r}\right) \Omega_{\alpha}\right)+\left(\phi\left(\bar{h}_{1}\right) \cdots \phi\left(\bar{h}_{r}\right) \Omega_{\bar{\beta}}, \frac{\Omega_{\alpha}-\Omega_{\beta}}{\alpha-\beta}\right)
$$

that the analyticity of $W_{\beta}$ follows from that of $\Omega_{\beta}$ and the strong continuity in $\beta$ of $\Phi_{\beta}=\phi\left(h_{1}\right) \cdots \phi\left(h_{r}\right) \Omega_{\beta}$. This latter fact can be seen either (incompletely) from higher order estimates, or from (iv)-(vi) of $\$ 2$. For by (vi) the projection onto $\Omega_{\beta}$ is given for small $|\beta|$ by the integral

$$
P_{\beta}=-\frac{1}{2 \pi i} \oint_{|\lambda|=\mu} R_{\beta}(\lambda) d \lambda
$$

with $0<\mu<m$. $\Omega_{\beta}=P_{\beta} \Omega_{0} /\left(\Omega_{0}, P_{\beta} \Omega_{0}\right)^{1 / 2}$. By (iv), $P_{\beta}$ is $L^{p}$ norm continuous in $\beta$ where $p \in I(\pi / 2-\varepsilon)$ of (2.9). But the product $\phi\left(h_{1}\right) \cdots \phi\left(h_{r}\right)$ is a multiplication operator which is in all $L^{q}, q<\infty$. Hence $\Phi_{\beta}$ is $L^{p}$ continuous. 
As in the above theorem we take $\beta$ in the sector $|\arg \beta| \leqq \pi-\varepsilon$ and $p \in I(\pi / 2-\varepsilon)$ where $\varepsilon>0$. Consider the expansion

$$
\begin{aligned}
R_{\beta}(\lambda) \Psi=\sum_{n=0}^{N}(-\beta)^{n}\left[\left(H_{0}-\lambda\right)^{-1} H_{I}\right]^{n}\left(H_{0}-\lambda\right)^{-1} \Psi \\
\\
+(-\beta)^{N+1} R_{\beta}(\lambda)\left[H_{I}\left(H_{0}-\lambda\right)^{-1}\right]^{N+1} \Psi .
\end{aligned}
$$

According to general asymptotic theory (cf. [4, p. 439-451]), the expansion (4.3) is asymptotic in $L^{p}$ provided all the terms $\left[\left(H_{0}-\lambda\right)^{-1} H_{I}\right]^{n}\left(H_{0}-\lambda\right)^{-1} \Psi$ make sense and $\lambda$ is in

$$
\left\{\lambda \mid \limsup _{\beta \rightarrow 0}\left\|R_{\beta}(\lambda)\right\|_{p}<\infty\right\}
$$

By the results of $\S 2$ this is certainly the case when $\Psi$ is in $L^{\infty}(M)$ and $\operatorname{Re}\left(\lambda e^{i \theta}\right)$ is sufficiently negative for $z=t e^{i \theta}$ in the cone (2.11).

By using the norm resolvent convergence $(v)$ of $\$ 2$ and the consequent stability of $E_{0}$, we obtain a somewhat stronger result:

THEOREM 4.2. Let $\varepsilon>0$ and suppose that $|\arg \beta| \leqq \pi-\varepsilon$ and $p \in I(\pi / 2-\varepsilon)$. For some $q>p$, let $\Psi \in L^{q}(M)$ and $\lambda \in \operatorname{res}\left(H_{0} \backslash L^{p}\right) \cap \operatorname{res}\left(H_{0} \backslash L^{q}\right)$. Then for sufficiently small $|\beta|, \lambda \in \operatorname{res}\left(H_{\beta} \mid L^{p}\right)$ and the series (4.3) for $R_{\beta}(\lambda) \Psi$ is asymptotic in $L^{p}$ as $\beta \rightarrow 0$. The series obtained for $P_{\beta}, \Omega_{\beta}, E_{\beta}$, and $W_{\beta}$ by inserting (4.3) into (4.2) are all asymptotic as $\beta \rightarrow 0$.

REMARKS. 1. The asymptotic series obtained for $E_{\beta}$ and $\Omega_{\beta}$ are just the RayleighSchrödinger series, and the series for $E_{\beta}$ can be expressed by a set of Feynman diagrams.

2. In particular we can take $\Psi \in D$ which is contained in $L^{q}(M)$ for all $q<\infty$ [13, Theorem III.9]. For $p=2$ we can also choose $\Psi \in C^{\infty}(N)$.

Proof. The fact that $\lambda \in \operatorname{res}\left(H_{\beta}\left\lceil L^{p}\right)\right.$ for sufficiently small $\beta$ follows from standard theory (cf. $[4$, p. 206]) and the norm resolvent convergence (v) of $\$ 2$. To prove that the remainder term in (4.3) is $L^{p}$-bounded we note that, by interpolation, $\left(H_{0}-\lambda\right)^{-1}$ is a bounded operator on $L^{r}$ for $r \in[p, q]$. Moreover, since $H_{I} \in L^{s}$ for all $s<\infty$ we see by Hölder's inequality that $H_{I}$ maps $L^{r}$ into $L^{r-\delta}$ for any $\delta>0$. Hence $\left[\left(H_{0}-\lambda\right)^{-1} H_{I}\right]^{n}\left(H_{0}-\lambda\right)^{-1} \Psi \in L^{p}$ for all $n$. The rest of the theorem is immediate.

We have been unable to apply our methods satisfactorily to time-dependent quantities such as the fields

$$
\phi_{\beta}(h, t)=\exp \left(i t H_{\beta}\right) \phi(h) \exp \left(-i t H_{\beta}\right) .
$$

The difficulty is that the operator $\exp \left(-z H_{\beta}\right)$ is well defined for $z$ in the cone (2.11) which does not include $z= \pm i$ when $\beta$ is nonreal. In fact, as $\arg \beta \rightarrow \pi$, $\exp \left(-z H_{\beta}\right)$ is defined only for $z=-i t+0$, and, as $\arg \beta \rightarrow-\pi$, for $z=i t+0$, where $t>0$. Accordingly, we see that our methods could be applied to objects of the form

$$
T_{\beta}=\left(\Omega_{\bar{\beta}}, \phi\left(h_{1}\right) \exp \left(i H_{\beta}\left(t_{1}+i \varepsilon_{1}\right)\right) \phi\left(h_{2}\right) \exp \left(i H_{\beta}\left(t_{2}+i \varepsilon_{2}\right)\right) \cdots \phi\left(h_{r}\right) \Omega_{\beta}\right)
$$


where $\varepsilon_{1}, \ldots, \varepsilon_{r}>0$ and $t_{1}, \ldots, t_{r} \gtrless 0$ depending on whether $\arg \beta \gtrless 0 . T_{\beta}$ can be regarded as a time-ordered VEV continued to the forward tube. However, at the present stage of the real $\beta$ theory, only the time-ordered VEV's with $r \leqq 4$ are known to exist as tempered distributions [9].

5. Borel summability. In this final section, we prove that for the $\left(\phi^{4}\right)_{2}$ theory the functions $W_{\beta}$ introduced by (4.1) can be recovered from their perturbation series by the method of Borel summability. Specifically, let $h_{1}, \ldots, h_{r}$ in $L^{2}(\mathscr{R})$ be given along with $\varepsilon>0$. Let $W_{\beta}$ have $\sum_{n=0}^{\infty} a_{n} \beta^{n}$ as asymptotic series. Then there exist a $B>0$ and $a>0$ such that

(i) $g(z)=\sum_{n=0}^{\infty}\left(a_{n} / n !\right) z^{n}$ converges if $|z|<a$ and has an analytic continuation to the sector $|\arg z|<\pi / 2$;

(ii) if $|\arg \beta|<\pi / 2-\varepsilon,|\beta|<B$, then $\int_{0}^{\infty} g(x \beta) e^{-x} d x$ exists;

(iii) $W_{\beta}=\int_{0}^{\infty} g(x \beta) e^{-x} d x$.

The Borel summability (i)-(iii) of $W_{\beta}$ complements that of the ground state energy, $E_{\beta}$, proven in [11]. It brings us one step closer to the scattering matrix which is expressed in terms of time-ordered unequal time vacuum expectation values.

By Watson's Theorem [3], to prove (i)-(iii) above, it is sufficient to prove that the remainder $\left|r_{n}(\beta)\right| \leqq A \sigma^{n} n !|\beta|^{n}$ for all $\beta$ with $|\beta|<B,|\arg \beta|<\pi-\varepsilon$ where $A$ and $B$ are ( $\varepsilon$-dependent) constants and $r_{n}(\beta)=W_{\beta}-\sum_{m=0}^{n-1} a_{m} \beta^{m}$. As usual we treat the remainders of the numerator and denominator of

$$
W_{\beta}=\left(\Omega_{0}, P_{\beta} \phi\left(h_{1}\right) \cdots \phi\left(h_{r}\right) P_{\beta} \Omega_{0}\right) /\left(\Omega_{0}, P_{\beta} \Omega_{0}\right)
$$

separately. Since the denominator has already been discussed in [11], we need only deal with the numerator. Finally, by (4.2), it is enough to bound the remainder of

$$
\left(\Omega_{0}, R_{\beta}\left(\lambda^{\prime}\right) \phi\left(h_{1}\right) \cdots \phi\left(h_{r}\right) R_{\beta}(\lambda) \Omega_{0}\right)
$$

uniformly in $\lambda, \lambda^{\prime}$ with $|\lambda|=\left|\lambda^{\prime}\right|=\mu$.

By using (4.3), one finds the remainder term for (5.1) is given by $\tilde{r}_{n}(\beta)$ $=(-\beta)^{n} \sum_{k=0}^{n} c_{k}$ where

$$
c_{k}=\left(\Omega_{0},\left[R_{0}\left(\lambda^{\prime}\right) H_{I}\right]^{n-k} R_{\beta}\left(\lambda^{\prime}\right) \phi\left(h_{1}\right) \cdots \phi\left(h_{r}\right) R_{\beta}(\lambda)\left[H_{I} R_{0}(\lambda)\right]^{k} \Omega_{0}\right) .
$$

Since $A \sigma^{n}(n+1) ! \leqq A(2 \sigma)^{n} n$ !, it is enough to obtain a bound of the form $A \sigma^{n} n$ ! on each $c_{k}$. By (3.21), in the sector of interest in $\beta,(N+1)^{s} R_{\beta}(\lambda)(N+1)^{-s+1}$ is bounded uniformly in $\lambda$. Taking $s=[(r+1) / 2]$ we see that

$$
\begin{aligned}
\left|c_{k}\right| \leqq & \left\|R_{\beta}\left(\lambda^{\prime}\right)\right\|\left\|\phi\left(h_{1}\right) \cdots \phi\left(h_{r}\right)(N+1)^{-s}\right\|\left\|(N+1)^{s} R_{\beta}(\lambda)(N+1)^{-s+1}\right\| \\
& \times\left\|\left[H_{I} R_{0}\left(\lambda^{\prime}\right)\right]^{n-k} \Omega_{0}\right\|\left\|(N+1)^{s-1}\left[H_{I} R_{0}(\lambda)\right]^{k} \Omega_{0}\right\| .
\end{aligned}
$$

The first three terms on the right side of (5.2) are independent of $n$ and are bounded uniformly in $\lambda, \lambda^{\prime}$ and $\beta$ with $|\beta|$ small, $|\arg \beta|<\pi-\varepsilon$. As in [11], the next terms are 
bounded by $A \sigma^{n-k}(n-k)$ ! and $A \sigma^{k}(k+s)$ ! where $A$ and $\sigma$ are independent of $\lambda, \lambda^{\prime}, n$ and $k$. Since

$$
(k+s) !(n-k) ! \leqq(n+s) !=\left(\begin{array}{c}
n+s \\
n
\end{array}\right) n ! s ! \leqq 2^{n+s} n ! s !
$$

we see the right-hand side of (5.2) is bounded by $C(2 \sigma)^{n} n$ ! where $C$ is independent of $\lambda, \lambda^{\prime}$ and $\beta$ satisfying the required conditions. This completes the proof of the bound on the remainder term for $W_{\beta}$. We have thus proved

THEOREM 5.1. Let $H_{I}=: \phi^{4}(g):$. For $h_{1}, \ldots, h_{r} \in L^{2}(\mathscr{R})$ let

$$
W_{B}=\left(\Omega_{\bar{\beta}}, \phi\left(h_{1}\right) \cdots \phi\left(h_{r}\right) \Omega_{\beta}\right)
$$

where $\Omega_{\beta}$ is for $\beta>0$ the vacuum vector for $H_{0}+\beta H_{I}$ normalized by $\left\|\Omega_{\beta}\right\|=1$, $\left\langle\Omega_{\beta}, \Omega_{0}\right\rangle>0$. Then the asymptotic series for $W_{\beta}$ is Borel summable to $W_{\beta}$.

\section{REFERENCES}

1. J. Glimm and A. Jaffe, $A \lambda \phi^{4}$ quantum field theory without cutoffs. I, Phys. Rev. (2) 176 (1968), 1945-1951. MR 40 \#1106.

2. - Field theory models, Les Houches Lectures, Gordon and Breach, New York, 1971.

3. G. Hardy, Divergent series, Clarendon Press, Oxford, 1949. MR 11, 25.

4. T. Kato, Perturbation theory for linear operators, Die Grundlehren der math. Wissenschaften, Band 132, Springer-Verlag, New York, 1966. MR 34 \#3324.

5. J. Lions, Espaces d'interpolation et domaines de puissances fractionnaires d'opérateurs, J. Math. Soc. Japan 14 (1962), 233-241. MR 27 \#2850.

6. A. McIntosh, On the comparability of $A^{1 / 2}$ and $\left(A^{*}\right)^{1 / 2}$, MacQuarie University, Sydney, Australia (preprint).

7. E. Nelson, A quartic interaction in two dimensions, Math. Theory of Elementary Particles (Proc. Conf., Dedham, Mass., 1965), M.I.T. Press, Cambridge, Mass., 1966. MR 35 \#1309.

8. L. Rosen, $A \lambda \phi^{2 n}$ field theory without cutoffs, Comm. Math. Phys. 16 (1970), 157-183.

9. - The $\left(\phi^{2 n}\right)_{2}$ quantum field theory: higher order estimates, Comm. Pure Appl. Math. 24 (1971), 417-457.

10. I. Segal, Construction of non-linear local quantum processes. I, Ann. of Math. (2) 92 (1970), 463-481.

11. B. Simon, Borel summability of the ground-state energy in spatially cutoff $\left(\phi^{4}\right)_{2}$, Phys. Rev. Lett. 25 (1970), 1583-1586.

12. - Coupling constant analyticity for the anharmonic oscillator, Ann. Physics 58 (1970), 76-136.

13. B. Simon and R. Høegh-Krohn, Hypercontractive semigroups and two dimensional selfcoupled Bose fields, J. Functional Analysis (to appear).

14. E. Stein, Topics in harmonic analysis related to the Littlewood-Paley theory, Ann. of Math. Studies, no. 63, Princeton Univ. Press, Princeton, N. J., 1970.

Department of Mathematics, University of California, Berkeley, California 94720

Departments of Mathematics and Physics, Princeton University, Princeton, New JERSEY 08540*

\footnotetext{
* Current address of both authors.
} 